\title{
EPIGENETIC CHANGES OF DRD2 GENE IN PATHOGENESIS OF SCHIZOPHRENIA
}

Nour El Huda Abd Rahim ${ }^{1}$, Mohd Nabil Fikri Rahim ${ }^{1}$, Norsidah Ku Zaifah ${ }^{1}$, Hanisah Mohd Noor $^{2}$, Kartini Abdullah ${ }^{2}$ and Norlelawati A. Talib ${ }^{3}$

${ }^{1}$ Department of Basic Medical Sciences, Kulliyyah of Medicine, International Islamic University Malaysia, Pahang, Malaysia, ${ }^{2}$ Department of Psychiatry, Kulliyyah of Medicine, International Islamic University Malaysia, Pahang, Malaysia, ${ }^{3}$ Department of Pathology and Laboratory Medicine, Kulliyyah of Medicine, International Islamic University Malaysia, Pahang, Malaysia.

Presenter: Nour El Huda Abd Rahim, elhuda@iium.edu.my

Introduction: The dopamine hypothesis has earlier dominated the theories for the development of schizophrenia based on the early pharmacologic evidence. The antipsychotic drugs, among others, is thought to interfere with the function of the dopamine D2 receptor (DRD2) resulting in clinical improvement. Accumulating evidence suggest the role of epigenetic mechanisms in the pathophysiology of schizophrenia. Despite this, specific evidence linking the DRD2 DNA methylation with schizophrenia is insufficient mainly due to the poor accessibility and limited brain samples. Of late, new data has suggested the global impact of DNA methylation in the development of schizophrenia, thus methylation in the peripheral blood could infer changes in the brain. The aim of this study was to assess the DRD2 DNA methylation in the peripheral blood of schizophrenia.

Materials and method: The case control study consisted of 138 schizophrenia patients and 132 healthy controls. The genomic DNA from the peripheral blood was bisulfite converted. The DRD2 DNA methylation level was quantitatively measured by using the MethyLight Taqman $\otimes$ assay and normalized with the ALU reference control to give the percentage methylation ratio. The demographic data was calculated using descriptive statistics while parametric variables were compared using independent samples $t$-test or analysis of covariance.

Results: There was a significant hypomethylation of DRD2 in schizophrenia compared to the control group $(p=0.001)$. The hypomethylation was also significant in males $(p=0.007)$ and females $(p=0.036)$ patients.

Conclusion: This study supports the possible role of DNA methylation of DRD2 gene could contribute to the pathogenesis of schizophrenia. 